G.A. Vliegenthart

H.N.W. Lekkerkerker

\section{Measurement of the interfacial tension of demixed colloid-polymer suspensions}

in cyclohexane a value of $1.5 \times$

$10^{-2} \mathrm{mN} / \mathrm{m}$ is obtained.

Key words Interfacial tension demixed suspensions - colloidpolymer mixtures

\begin{abstract}
The interfacial tension of a demixed colloid-polymer mixture is measured using the spinning drop technique. For a demixed system of colloidal silica (diameter $20 \mathrm{~nm}$ ) and polydimethylsiloxane $\left(M_{w}=97000\right)$
\end{abstract}

Dr. G.A. Vliegenthart $(\bowtie)$

H.N.W. Lekkerkerker

Van't Hoff Laboratory

for Physical and Colloid Chemistry

Utrecht University

Padualaan 8

P.O. Box 80.015

3508 TB Utrecht, The Netherlands and colloid and the interface has a thickness of at least one colloidal diameter which makes it thick compared to interfaces in molecular systems. A first estimate of the interfacial tension in this system is then provided by the scaling relation $k T / \sigma^{2}$ where $\sigma$ denotes the diameter of the colloidal particle. For a particle diameter $\sigma=20 \mathrm{~nm}$, one therefore expects a value of $0.01 \mathrm{mN} / \mathrm{m}$.

In this article we will present results of first measurements of the interfacial tension of a demixed colloid-polymer mixture. The measurements have been done using the spinning drop technique $[16-18,9]$ on a mixture of sterically stabilized silica and the polymer polydimethylsiloxane in cyclohexane.

divided by an interface. The nature of the coexisting phases depends on the size ratio of the polymers and the colloids [3]. For size ratios larger than $R_{\mathrm{g}} / r_{\mathrm{c}}=0.3\left(R_{\mathrm{g}}\right.$ is the radius of gyration of the polymer and $r_{\mathrm{c}}$ is the radius of the colloid) and moderate colloidal volume fractions one finds coexistence between a colloidal-gas and a colloidal-liquid phase as is predicted by the theory [3-5] and confirmed by experiments [3, 4, 6-8].

In contrast to demixed colloid polymer suspensions the interfacial properties in demixed polymer solutions $[9,10,12,13]$ and in complex coacervates and their equilibrium liquid [11] have been studied. In these systems one finds typical values for the interfacial tension in the range $0.01-0.001 \mathrm{mN} / \mathrm{m}$. The interface in demixed colloid polymer mixtures is also an interesting object to study. The coexisting phases only differ in concentration of polymer

\section{Experimental}

\section{System}

The system we studied consisted of a mixture of colloidal silica spheres (stearyl-coated Ludox HS40, Dupont, $r_{\mathrm{c}}=10 \mathrm{~nm}(\mathrm{TEM})$, polydispersity $10 \%$ ) and polymer coils (PolyDiMethylSiloxane (PDMS, Janssen Chimica) of mol.wt. $97000 \mathrm{~g} / \mathrm{mol}, R_{\mathrm{g}}=14 \mathrm{~nm}$ [15]) dissolved in cyclohexane (Janssen). All components have a refractive index of 1.44 differing at most 0.005 making the solutions fully transparent. 
Phase diagram

The phase diagram of this mixture is determined using the method of dilution lines. A series of mixtures was prepared with different compositions of colloid and polymer. These were then diluted or concentrated by adding or evaporating cyclohexane until the phase boundary was crossed. In Fig. 1 we present the phase diagram as determined by visual observation. The phase separation always resulted in two phases separated by a very sharp and flat interface on which surface waves could easily be excited by just tilting the tube. These are indications that the interfacial tension is low. The spinning drop experiments discussed below have been done in the mixture indicated by the black dot in Fig. 1.

To calculate the interfacial tension the density difference between the coexisting phases is needed. Because of a lack of material the density difference was not measured but estimated from the phase diagram. To make this estimate we have to make only one assumption, that is that all the colloid is in the dense phase and all the polymer is in the dilute phase. Knowing the overall composition, the density of silica $(1.791 \mathrm{~g} / \mathrm{ml})$, the density of PDMS $(0.774 \mathrm{~g} / \mathrm{ml})$, the density of cyclohexane $(0.994 \mathrm{~g} / \mathrm{ml})$ and that the volume of the upperphase is $1 / 15$ of the total volume, we calculate $\Delta \rho$ to be $300 \mathrm{mg} / \mathrm{ml}$. The actual value might be somewhat smaller because some colloid will be in the upper phase and some of the polymer will be in the lower phase.

\section{Spinning drop}

To measure the interfacial tension we used a home-built spinning drop tensiometer [14]. The spinning drop tube

Fig. 1 Phase diagram of the colloid polymer mixture of size ratio $R_{\mathrm{g}} / r_{\mathrm{c}}=1.4$. The black dot corresponds to the composition at which the spinning drop experiments were done

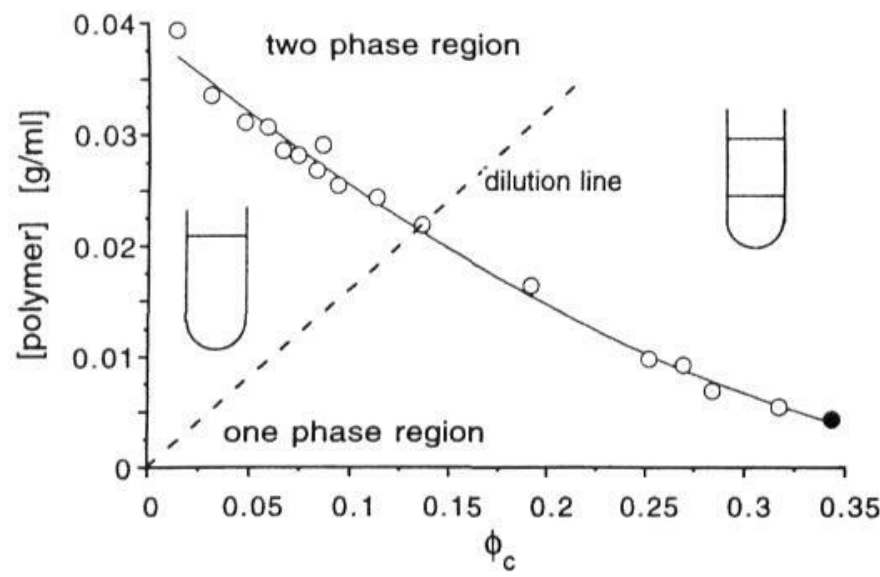

was made of Wilmad preciscion bore glass, internal diameter $3.96 \mathrm{~mm}$, length $31.4 \mathrm{~mm}$ and was sealed with Teflon stops. The angular velocity was measured using an optical sensor. The droplet was observed through a microscope and its length and diameter were measured with a crosswire and a micrometer.

The samples were prepared by first filling the tube with the dense phase after which a small drop of the less-dense phase was added with a glass capillary. By rotating at high $\omega$, an elongated thread of the dilute phase was formed and centered on the rotational axis. By decreasing $\omega$ this thread broke up into individual droplets. The angular velocities at which the measurements were done were in the range of $50<\omega<250[\mathrm{rad} / \mathrm{s}]$. After allowing the system to equilibrate for $1-2 \mathrm{~h}$ the length and diameter were measured. All the measurements were carried out at $25^{\circ} \mathrm{C}$.

The volume of the dilute phase was found to be critical for the formation of droplets. When this volume was too big, all sorts of irregular axial symmetric droplet shapes developed. These shapes did not show any relaxation towards more symmetrical shapes. The evaporation of cyclohexane, limited the equilibration time to $1-2 \mathrm{~h}$. The formation of air bubbles made further measurements then impossible. In spinning drop experiments this equilibration time is often $6-8 \mathrm{~h}$.

\section{Results and discussion}

The theory derived by Princen et al. [16] was used to calculate the interfacial tension. In this theory the shape of a droplet is calculated by balancing the Laplace pressure over the interface and the centrifugal pressure over the interface. The parameters needed to do the calculations are the length and diameter of the droplet (measured), $\omega$ (measured) and the density difference between the coexisting phases (estimated, see experimental).

Two photographs of droplets formed in the spinning drop tube are shown in Fig. 2.

In Fig. 3 we present the values of $\gamma / \Delta \rho$ at different angular velocities. There seems to be a trend that $\gamma / \Delta \rho$ decreases as $\omega$ increases. This can be a consequence of the fact that not enough points have been measured at high (1) to gain good statistics.

Averaging the values from Fig. 3 we find $\gamma / \Delta \rho=$ $5 \times 10^{-8}\left[\mathrm{~m}^{3} / \mathrm{s}^{2}\right]$. The value of $\gamma$ we then calculate is $\gamma=0.015 \mathrm{mN} / \mathrm{m}$. The experimental value of $\gamma$ and the value from the simple scaling argument given in the Introduction are in good agreement.

Recently, Vrij [20] has adapted the statistical mechanical perturbation theory of Kalikmanov and Hofmans for planar liquid-vapor interfaces [19] to calculate the 
Fig. 2 Two droplets formed in the spinning drop tube. A The length of this droplet was about $1.9 \mathrm{~mm}$ and the angular velocity was $152 \mathrm{rad} / \mathrm{s}$; B The length of this droplet was $1.4 \mathrm{~mm}$, the angular velocity was $248 \mathrm{rad} / \mathrm{s}$. The contrast between in and outside of the droplet is low because of the matching of the refractive index

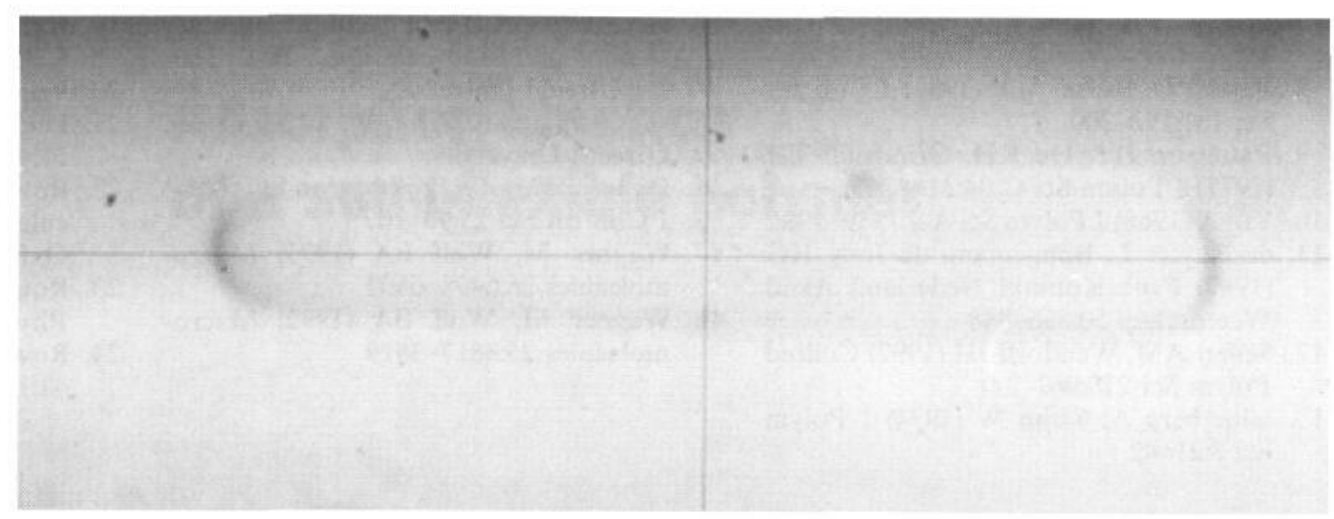

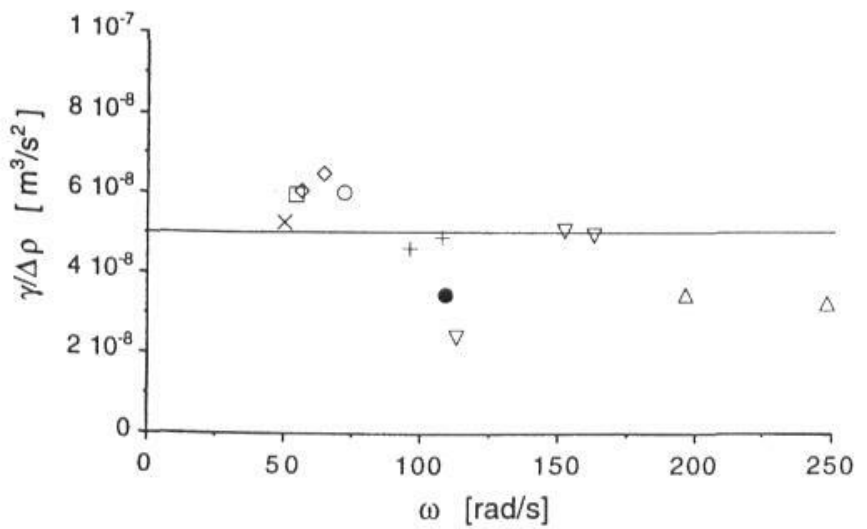

Fig. $3 \gamma / \Delta \rho$ as a function of (1). Points having the same symbol correspond to droplets measured at different rotational speeds. The point indicated by the open triangle at $\omega=250[\mathrm{rad} / \mathrm{s}]$ corresponds to the droplet from Fig. 2a, the one indicated by an open triangle at $\omega=150[\mathrm{rad} / \mathrm{s}]$ corresponds to the droplet in Fig. 2b

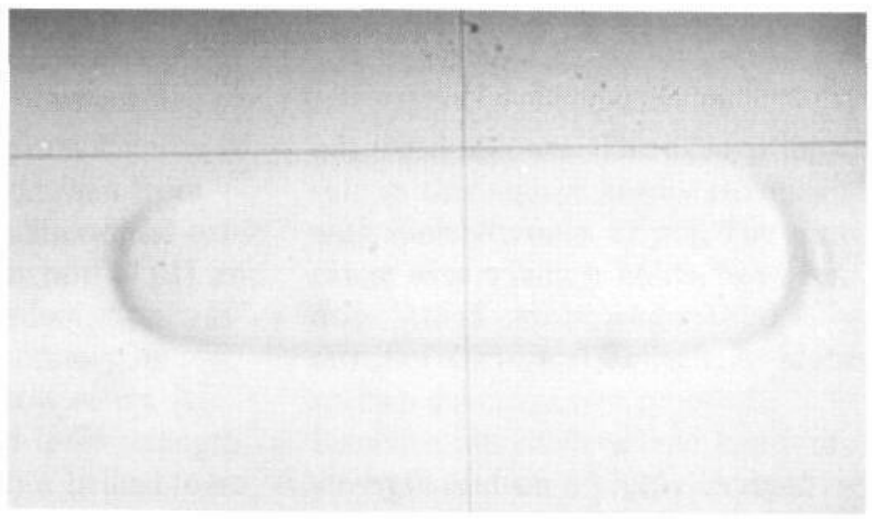

interfacial tension in demixed colloid polymer suspensions. For the case that $R_{\mathrm{g}} / r_{\mathrm{c}}=1, \phi_{\mathrm{c}}=0.325$ and a polymer volume fraction $(N / V) \frac{4}{3} \pi R_{\mathrm{g}}^{3}=0.55$ Vrij [20] finds a value of $0.005 \mathrm{mN} / \mathrm{m}$.

At this moment there is no theory for the interfacial free energy and the concentration profile for demixed colloid polymer mixtures. Like in the case of the phase diagram $[5,21]$ the non-additive hard sphere of Widom and Rowlinson [22-24] may provide a good starting point for such a theory.

Further investigations, both theoretical and experimental, are required to improve the understanding of the interfacial properties of demixed colloid polymer mixtures.

Acknowledgment The authors are grateful to C.J. Rietveld. I.C.J. Dur, B.W.M. Kuipers and J. Suurmond for their technical support.

\section{References}

1. Asakura S, Oosawa F (1954) J Chem Phys 22:1255 1256

2. Vrij A (1976) Pure \& Appl Chem 48:471- 483
3. Gast AP, Hall CK, Russel WB (1983) J Colloid Interface Sci 96:251-267

4. Vincent BJ, Edwards J. Emmet S, Croot R (1988) Colloid Surf 31:267 294
5. Lekkerkerker HNW, Poon WCK, Pusey PN, Stroobants A. Warren P (1992) Europhys Lett 20:559-564

6. de Hek H, Vrij A (1981) J Coll Int Sci 84:409-422 
7. Sperry PR (1984) J Coll Int Sci 99:97-108

8. Patel PD, Russel WB (1989) J Coll Int Sci 131:193-200

9. Patterson HT, Hu KH, Grindstaff TH (1971) J Polym Sci C 34:31-43

10. Vrij A (1968) J Polym Sci A-2:1919-1932

11. de Ruyter L, Bungenberg de Jong HG (1947) Proc Koninkl Nederland Akad Wetenschap 50:836-848

12. Seifert AM, Wendorff JH (1992) Colloid Polym Sci 270:962 971

13. Silberberg A, Kuhn W (1934) J Polym Sci $8: 21-42$
14. van Aken GA (1990) A study of Winsor II microemulsion equilibria. PhD Thesis, Utrecht University

15. van der Zande B (1995) MSc Thesis, Utrecht University

16. Princen HM, Zia IYZ, Mason SG (1967) J Coll Int Sci 23:99-107

17. Wagner M, Wolf BA (1993) Macromolecules 26:6498-6502

18. Wagner M, Wolf BA (1992) Macromolecules 25:3817-3819
19. Kalikmanov VI, Hofmans GCJ (1994) J Phys Condens Matter 6:2207-2214

20. Vrij A (1997) Physica A 235:120-128

21. Lekkerkerker HNW (1990) Coll Surf $51: 419-426$

22. Rowlinson JS, Widom B (1982) Molecular Theory of Capillarity, Chap 5. Oxford: Clarendon Press

23. Rowlinson JS, Widom B (1970) J Chem Phys 52:1670

24. Rowlinson JS (1980) Adv Chem Phys $41: 1$ 